\title{
Reflexiones para una práctica docente de integración de destrezas y contenidos en los programas de español a nivel universitario
}

\author{
Kari Soriano Salkjelsvik
}

Universidad de Bergen

\section{SUMMARY}

This article is a reflection on the possibilities that the concept of Content and Language Integrated Learning (CLIL), can provide for the teaching of foreign language programs at the university level, using as an example the programs of Hispanic studies in Norway. After reviewing the concept of CLIL, and placing it in the context of Hispanic studies, the article investigates its practical, political, and educational applications. It is argued that integrating skills and content fully at the university level is a task that depends on specific administrative infrastructures, as well as on methodological resources that can make this integration an object of study and reflection. In this context, this work aims to promote the creation of new teaching proposals for university programs of foreign languages that respond to the challenges and demands that are currently facing the humanities.

\section{RESUMEN}

Este artículo se concibe como una reflexión sobre las posibilidades que el concepto de Aprendizaje integrado de contenido y lengua (AICLE), puede aportar para la enseñanza los programas de lenguas extranjeras en las universidades, utilizando como ejemplo los programas de estudios hispánicos en Noruega. Tras revisar el concepto de CLIL, y situarlo en el contexto de los estudios hispánicos, el artículo indaga sobre su aplicación práctica y político-educativa. Se arguye que integrar destrezas y contenidos plenamente a nivel universitario es una tarea que depende de infraestructuras administrativas específicas, así como de recursos metodológicos que hagan de dicha integración un objeto de estudio y reflexión. En este sentido, este trabajo quiere impulsar la creación de nuevas propuestas docentes para los programas universitarios de lenguas extranjeras que respondan a los retos y exigencias a los que actualmente se están enfrentando las humanidades. 


\section{INTRODUCCIÓN}

Durante los últimos 20 años, las aportaciones pedagógicas y didácticas para la clase de lenguas extranjeras han ido apuntando, cada vez más, hacia la necesidad de integrar destrezas y contenidos, para así promover conjuntamente en el aula el desarrollo y la consolidación de las diferentes competencias lingüísticas del estudiante en la lengua meta y el aprendizaje de contenidos académicos específicos. ${ }^{1}$ Normalmente, la noción de integrar destrezas y contendidos se ha asociado con la enseñanza en las escuelas, sobre todo en las escuelas bilingües. No obstante, hay que recordar que, a nivel universitario, y en países que no son de habla hispana, el docente también ha utilizado el español regularmente para impartir las diferentes disciplinas que conforman los programas de filología hispánica² . ¿Es esta una práctica consciente de integración de destrezas y contenido, o se trata más bien de una fe ciega en que el estudiante, además de aprender el contenido, perfeccionará su competencia lingüística con solo seguir la clase en español y estudiar los materiales asignados?

Ante estas cuestiones, quiero proponer una meditación alrededor de lo que implicaría integrar de forma rigurosa destrezas y contenido en las clases universitarias de especialización en estudios hispánicos en contextos en los que el español es una lengua extranjera. Por ello, el presente trabajo es ante todo un texto reflexivo cuya meta es traer a colación algunos factores y precauciones a considerar en las evaluaciones de los cursos de especialización universitaria en filología hispánica. En particular, me gustaría incitar aquí a que los profesores universitarios de español revisen proactivamente el papel que juegan las destrezas en sus cursos. En otras palabras, como profesora universitaria lo que propongo es repensar qué es lo que entendemos por enseñar español, en su más amplio sentido, en la universidad y cómo lo hacemos.

\footnotetext{
${ }^{1}$ La noción de "contenido" es objeto de estudio y discusión entre pedagogos y didactas, y ha sido definida en diferentes términos. La acepción más extendida, y a la que subscribo este trabajo, sigue el estudio de J. Crandall y G. R. Tucker (1994), que entienden por "contenido" aquella materia que es claramente académica. Aunque hay que recordar que críticos como F. Genese (1994), P.P. Chaput (1993) o M. Met (1991) opinan que cualquier tema o material puede convertirse en "contenido" en la clase de competencia lingüística, ya que lo importante es la manera en la que se trabajan los materiales en clase, la relevancia que estos tienen para el crecimiento intelectual del estudiante y el apoyo que pueden ofrecer al desarrollo de la lengua meta.

${ }^{2}$ Utilizo el término filología hispánica en un sentido muy general para incluir los programas universitarios de grado en español y que incluyen diferentes disciplinas de temas del mundo hispánico; siendo las más tradicionales la lingüística, la literatura, la traducción y la historia. Estos grados tienen nombres y composiciones disciplinarias diferentes en los distintos países.
} 


\subsection{La integración de destrezas y contenidos}

Los defensores de la integración de destrezas y contenido subrayan las ventajas de enseñar en la lengua meta materias escolares o académicas a estudiantes no nativos. Entre las virtudes de esta aproximación didáctica, se destacan, entre otras, que el estudiante se verá expuesto a un uso de la lengua extranjera en un contexto natural y comprensible (Krashen) y que de este modo aprenderá a comunicarse en diferentes registros. Es más, al verse expuesto a diferentes materiales auténticos en la lengua meta, el estudiante se adentrará en un contexto cultural más amplio y rico que el presentado en los libros de texto tradicionales para la enseñanza de destrezas, además de verse expuesto a diferentes métodos de enseñanza. Se trata, por tanto, de facilitar un complejo proceso en el que el alumno interpreta la lengua extranjera, a la vez que asimila un nuevo conocimiento sobre el contenido académico impartido. En este contexto, el alumno se verá también motivado por los retos cognitivos que le ofrecen tanto el contenido como las destrezas, por lo que su aprendizaje será más efectivo (Lightbown y Spada).

Finalmente, se subraya que la integración de destrezas y contenido promoverá la competencia intercultural del alumno y lo preparará mejor para la vida laboral y/o futuros estudios.

No obstante, hay críticos que cuestionan que la integración de destrezas y contenidos sea válida para todos los contextos. Anthony Bruton (2011), por ejemplo, mantiene que la enseñanza basada en la integración de destrezas y contenidos no es tan beneficiosa como proponen sus defensores y demanda más estudios sobre el impacto que dicha práctica tiene sobre la metodología y el currículo, así como sobre la influencia que este acercamiento tiene sobre los estudiantes no necesariamente sobresalientes. El argumento de Bruton es que la integración de contenidos no se puede poner en práctica más que en contextos didácticos muy específicos e incluso selectos. ${ }^{3}$ A pesar de las críticas, actualmente parece haberse convertido en un lugar común afirmar que todo alumno o estudiante se puede beneficiar de una enseñanza en la que un contenido académico específico (matemáticas o geografía, por ejemplo) se imparta en la lengua meta (el español, como es nuestro caso). ${ }^{4}$

\footnotetext{
${ }^{3}$ Ver también el artículo de Ioannou Georgiou (2012) y el estudio de Harrop (2012).

${ }^{4}$ En este trabajo me referiré al español estándar (castellano y todas la variantes tanto de España como de Hispanoamérica) utilizando el término "español". Esto lo hago por defecto y no por falta de respeto a las otras variedades. En el contexto universitario, los departamentos que enseñan cultura hispánica normalmente se acogen bajo nombres como Departamento de Español, Departamento de Lenguas Romances, Departamento de Estudios
} 


\subsection{La integración de destrezas y contenidos en español: tradición y terminologías}

En el mundo hispanohablante, la educación bilingüe ha sido discutida extensamente, ya desde el siglo XIX, en el contexto de las reformas educativas que trajo la Ilustración. ${ }^{5}$ Además, la enseñanza bilingüe ha tenido una larga tradición en nuestros países, ya que tanto en España como en Hispanoamérica el castellano convive con otras lenguas habladas a diario por los alumnos: a veces oficiales y otras no, unas veces de la misma familia que el castellano como el catalán o el gallego - pero la mayoría no, como el vasco o las numerosas lenguas indígenas de Hispanoamérica. Esto ha dado como resultado que exista un gran número de escuelas bilingües en el mundo hispano, así como una casi indiscutible fe en la superioridad de las mismas. ${ }^{6}$

Hoy en día, para referirse a los acercamientos que integran contenido y competencia lingüística en clase existen varias terminologías en español. Quizás las más utilizadas sean Enseñanza de lengua basada en contenidos o Enseñanza de lengua y contenidos integrados. En inglés también encontramos diferentes términos, entre ellos Content-Based Instruction (CBI), que es el más utilizado en el ámbito estadounidense y que D.M. Brinton define como ${ }^{7}$ :

the integration of content learning with language teaching aims. More specifically, $[\mathrm{CBI}]$ refers to the concurrent study of language and subject matter, with the form and sequence of language presentation dictated by content material. (vii)

\footnotetext{
Hispánicos, Departamento de Lenguas Extranjeras, etc. Una búsqueda rápida en Google, muestra solo un Departamento de Castellano, el de la Universidad Pedagógica de Chile (UMCE).

${ }^{5}$ La educación bilingüe durante el siglo XIX iba sobre todo destinada a la integración social de las minorías étnicas. Para Maria Luisa Pérez Gras (2010) el proyecto de educación bilingüe decimonónico fue un proyecto utópico, como demuestra en su estudio del caso argentino.

${ }^{6}$ Ahora bien, como señala Silvia Montrul, en este contexto es importante recordar que en el mundo hispanohablante coexisten dos tipos de bilingüismo: uno de la clase media y alta "que aprenden inglés u otra lengua europea con alcance global" (277) y el bilingüismo indígena o autónomo que no tiene el mismo prestigio.

${ }^{7}$ Como ya he mencionado, CIB es el acrónimo más usado en los Estados Unidos, aunque también encontramos otros términos quizás menos frecuentes, como Content Based Approach (CBA), que Madrid y García Sánchez definen como un acercamiento en el que "the TL (Target Language) is the means of instruction and is effectively taught through the medium of subject matter. The TL is viewed as the vehicle through which the curricular contents are taught and learnt, rather than as the object of study" (125).
} 


\section{Nordic Journal of Modern Language Methodology}

Desde un punto de vista teórico, los diferentes términos marcan ciertas variaciones metodológicas. ${ }^{8}$ Pero en Europa, y en los últimos años, se ha ido utilizando cada vez más el término Content and Language Integrated Learning (CLIL), que en español a veces se traduce a Aprendizaje integrado de contenido y lengua (AICLE), para agrupar la gran variedad terminológica que ha surgido alrededor de este concepto:

The acronym CLIL is used as a generic term to describe all types of provision in which a second language (a foreign, regional or minority language and/or another oficial state language) is used to teach certain subjects in the curriculum other that the language lessons themselves. (European Commision, 2006: 8)

Según esta definición general de la Comisión Europea, impartir contenidos en español en los programas universitarios de filología hispánica es por tanto una práctica CLIL. Por ende, todas estas propuestas arguyen que si se crea una práctica didáctica en la que se aplican acercamientos específicos de integración de destrezas y contenidos, el resultado será que los estudiantes mejorarán sus competencias lingüísticas —incluido un lenguaje académico (Cummins 1981) - a la vez que adquirirán conocimientos sobre contenidos académicos de manera óptima. En este contexto, lo que me importa en este momento no es tanto revisar y proponer metodologías, como reflexionar sobre el papel que juegan las destrezas en los cursos universitarios de especialización.

\subsection{Los programas de filología hispánica}

En Europa, es una práctica muy extendida impartir los cursos de especialización en filología hispánica en español. ${ }^{9}$ No obstante, las diferentes disciplinas que se imparten en estos programas han permanecido siempre separadas, incluso con la prevalencia durante los últimos años de los llamados estudios culturales. Por eso, observamos que los profesores de, por ejemplo, literatura, rara vez se aventuran a impartir materias como lingüística o didáctica.

\footnotetext{
${ }^{8}$ Existen una gran variedad de propuestas para la aplicación práctica de este tipo de enfoque integrado. Ver, por ejemplo, Baetens-Beardsmore (1993) o Marsh, Marjels y Hartiala (2001)

${ }^{9}$ Las excepciones más conocidas dentro del mundo académico son quizás Inglaterra y Rusia, donde es tradicional impartir las clases en la lengua nativa.
} 


\section{Nordic Journal of Modern Language Methodology}

Esto se debe a que estos académicos, desde el punto de vista de su formación e investigación, pertenecen a campos de estudio claramente diferenciados, con acercamientos metodológicos y teóricos distintos, y que dialogan, además, con tradiciones críticas bien diferentes. La idea es que la docencia en la universidad ha de ser impartida por especialistas en el campo, ya que ha de estar basada en la investigación del profesor.

Hay que recordar también que en los cursos de especialización, la programación de los cursos se desarrolla habitualmente alrededor de los temas tanto conceptuales como teóricos que cada disciplina define para su propio campo. Es decir, el centro de enfoque de cada clase es un contenido académico específico bien sea lingüístico, cultural, histórico o literario, por lo que muy rara vez se encuentran cursos en el currículum verdaderamente interdisciplinarios. Es más, en los cursos universitarios de especialización raramente se habla sobre las destrezas y se presupone que el estudiante ya posee cierto dominio del español antes de comenzar el programa, por lo que la perfección de sus diferentes competencias lingüísticas queda tradicionalmente relegada a un segundo plano. Incluso en las clases de lingüística española el enfoque recae sobre el meta-discurso lingüístico, hasta en los seminarios de prácticas, donde se suelen realizar ejercicios alrededor de estructuras lingüísticas específicas. Lo que presupone este sistema educativo es que el nivel de destreza lingüística del estudiante se seguirá desarrollando en los cursos universitarios de especialización si este atiende a las clases teóricas impartidas en español, lee textos en esta lengua y participa en las clases de prácticas sean sobre el tema que fueren. Siguiendo esta lógica, el perfeccionamiento de las destrezas ocurrirían de manera natural, e incluso, de manera inevitable.

\section{REFLEXIONES}

La pregunta que me hacía al principio de este trabajo, era si el profesor universitario en los programas de español es consciente de que su labor docente es una práctica CLIL o si cree que al impartir cursos de especialización en español el estudiante perfeccionará, como si fuera por ósmosis, sus destrezas en español. La pregunta es evidentemente retórica, pero me da pie para recapacitar sobre algunas de mis preocupaciones relacionadas con la cuestión de la integración de destrezas y contenidos a nivel universitario. 


\subsection{El gran salto}

Si pensamos en la instrucción del español a lo largo de todo el sistema educativo, desde que se empieza a impartir en la escuela como asignatura optativa hasta los cursos de grado universitarios, observamos que existe un gran salto metodológico y didáctico entre la escuela y la universidad. Pues, si bien en la escuela el enfoque incurre sobre todo en el desarrollo de las destrezas, en la universidad el peso recae, como ya discutimos, en los contenidos de las diferentes disciplinas. ${ }^{10}$

Esta muralla, aparentemente inamovible, se debe en parte a una cultura educativa que concive la educación universitaria en humanidades como una educación primordialmente teórica. Desde un punto de vista institucional, en los programas de lenguas extranjeras ha sido tradicionalmente importante marcar una diferencia clara entre la actividad docente de las universidades y la de las "academias de idiomas". Pero la realidad es que el papel de las humanidades en Europa está cambiando a grandes pasos ante la demanda social y política de crear programas educativos con una clara aplicación profesional e instrumental. Bien es sabido que las directrices de la Comisión Europea llevan ya insistiendo desde su comunicación Rethinking Education: Investing in skills for better socio-economic outcomes (2012) en la urgencia de modernizar los programas universitarios para que se adapten a las necesidades económicas europeas. Es más, en el Marco estratégico: Educación y Formación 2020 se especifica que las reformas universitarias han de realizarse "con el fin de formar titulados con cualificaciones de alto nivel adecuadas a la oferta de empleo y competencias transferibles que les preparen para los cambios del mercado laboral" (Comisión Europea "Calidad e idoneidad de la educación superior"). En Noruega, el reciente Mensaje de Estado 25 (2016-2017), el llamado Humaniorameldingen, se une a esta política europea al subrayar la necesidad de que los programas de lenguas extranjeras se adapten más a las necesidades del mercado: "Det er viktig å trekke inn arbeids- og næringslivet i arbeidet med fremmedspråk på alle nivåer i utdanningssystemet, både i forbindelse med rekruttering og tiltak for å synliggjøre relevansen av språkkunnskaper" ('Språk: kapittel 6).

\footnotetext{
${ }^{10}$ Los estudios sobre los libros de texto que se utilizan en el sistema escolar noruego muetran como los contenidos de tipo académico (culturales o literarios) tienen una función que se supedita a la enseñanza de las diferentes destrezas (Eide 2012, Olsbu y Salkjelsvik 2008, Olsbu 2014). El caso de Noruega es representativo en un contexto europeo.
} 


\section{Nordic Journal of Modern Language Methodology}

Resulta interesante que efectivamente estos objetivos de aprendizaje son los que a menudo aparecen descritos en los planes de estudio de los programas de español a nivel universitario en Noruega. Baste como ejemplo el programa de Bachelor en español y estudios latinoamericanos de mi universidad, la Universidad de Bergen, que define las metas de aprendizaje en los siguientes términos:

El estudio tiene unos objetivos tanto teóricos como prácticos: se facilitará la práctica de las habilidades lingüísticas y se desarrollará la comprensión de los fenómenos lingüísticos, culturales y literarios. De esta manera [el programa] es una disciplina instrumental para profesiones que exigen conocimientos sólidos sobre América Latina y es una base para continuar especializándose en los diferentes campos de investigación de los estudios latinoamericanos. (mi subrayado) ${ }^{11}$

En otras palabras, los objetivos del programa se precisan a la vez como teóricos y pragmáticos, pues han de satisfacer necesidades académicas y también profesionales: con estos estudios los estudiantes han de poder utilizar sus conocimientos tanto para salir al mercado laboral convencional, como para seguir preparándose para una carrera en la investigación si así lo prefirieran.

Ahora bien, en la práctica, los programas de lenguas extranjeras a nivel universitario son los que quizás más se resisten a integrar de manera concreta el desarrollo de las diferentes destrezas lingüísticas del estudiante; es decir, a fomentar el tipo de conocimiento más instrumental y transferible del aprendizaje de estos programas. En principio podría parecer innecesario crear actividades diseñadas específicamente para el desarrollo de las diferentes destrezas, pues los estudiantes ya llegan a la universidad con cierto nivel de español. Y bien es cierto que una vez el estudiante pasa a cursos universitarios cuyo enfoque es un contenido académico específico - ya sea literatura, historia, cultura, cine u otros - , sus diferentes destrezas - lectura, comunicación, intercomunicación, escritura y competencia auditiva - se

\footnotetext{
${ }^{11}$ Mi traducción de "Studiet har både eit praktisk og eit teoretisk siktemål: Det skal leggja til rette for å øva opp språklege evner og utvikla forståinga av lingvistiske, kulturelle og litterære fenomen. På den måten er det både reiskapsfag for utdanning til yrke som krev solide kunnskapar om Latin-Amerika, og eit grunnlag for vidare fordjuping innan dei ulike forskingsfelta i latinamerikastudiet." (http://www.uib.no/studieprogram/BAHFSPLA\#studieplan)
} 
seguirán consolidando y mejorarán si los cursos son impartidos en español. Esto es debido a que en este tipo de cursos el estudiante se ve expuesto a registros discursivos nuevos y a que con la práctica algo siempre se asimila de dichas competencias. Pero la simple exposición al español académico no garantiza que el estudiante aprenda a utilizarlo de manera efectiva. Por ello propongo, como ya sugerí, que dialoguemos sobre la manera en que los cursos de especialización atienden a la necesidad del estudiante de obtener conocimientos críticos y teóricos sobre las diferentes disciplinas, a la vez que sigue progresando en el desarrollo y consolidación de sus destrezas. La meta debe ser que el estudiante aprenda a relacionarse con sus conocimientos de una manera integral y a expresarse sobre ellos en un registro español adecuado a la situación en que lo emplea. En otras palabras, se trataría de pasar de una enseñanza aislada de contenidos a una enseñanza en la que se cree una relación simbiótica ente las destrezas, los contenidos y su transferibilidad.

Pero existen obstáculos. El primer punto de resistencia descansa sobre el hecho de que las propuestas didácticas sobre la enseñanza integrada, al estar diseñadas sobre todo para los ciclos escolares primario y secundario, no tienen en cuenta que en la universidad la lengua meta — en este caso el español — no es sólo un conjunto de destrezas a adquirir, sino también un objeto de estudio con contenidos propios. En este contexto, reducir la lengua a "un medio para el aprendizaje" es olvidar la tradición teórica y crítica de los estudios lingüísticos en general. Además, hay que recordar que gran parte de la tarea del profesor universitario es enseñar al estudiante a acceder al conocimiento implícito que ya posee sobre las estructuras lingüísticas que practica con sus destrezas. Pues en el momento en que el estudiante haga explícito este conocimiento teórico, se convertirá en un estudiante independiente y estará preparado para hacer sus propias reflexiones y aprender nuevas estructuras lingüísticas en español. Es decir, a nivel universitario se entiende que no se puede prescindir de la reflexión meta-lingüística o desatender a los lazos que existen entre ésta y el aprendizaje de las destrezas. Por ello, uno de los retos de la enseñanza del español en la universidad es la necesidad de hacer frente a diferentes objetivos de aprendizaje en una misma clase y en un tiempo limitado. De una parte, los estudiantes han de adquirir herramientas teóricas y metodológicas específicas de cada disciplina; de otra, deben continuar desarrollando sus destrezas para poder utilizar el español ya bien sea en el ámbito laboral o el académico. 
El segundo de los obstáculos es la resistencia que existe entre profesores universitarios en programas de lenguas extranjeras hacia el componente de utilidad que viene exigiendo la nueva política educativa europea en general, incluída la noruega. Por un lado, se entiende que la universidad debe ofrecer una instrucción en humanidades que sea ante todo teórica y especulativa. Por otro, se teme que el enfoque excesivo en el utilitarismo en la educación iría en detrimento de la importante labor de crítica y reflexión cultural e intercultural que tradicionalmente tiene lugar desde las humanidades. No obstante, el cambio, como vimos, ya está aquí y requiere transformaciones estructurales en la manera que organizamos nuestra docencia. Lo importante, por ello, es que las reformas de nuestros programas de español se hagan de manera que la dimensión utilitaria de la enseñanza fortalezca los contenidos de las disciplinas, no que les haga ceder terreno.

Es mi firme creencia que los objetivos prácticos y teóricos definidos en los planes de estudio de los programas de español a nivel universitario no se pueden alcanzar sin un acercamiento holístico a la tarea docente del español, un acercamiento que ofrezca experiencias de aprendizaje que ayuden a comprender las relaciones conceptuales que existen entre las destrezas y los diferentes contenidos académicos del programa.

\section{INTEGRACIÓN O COEXISTENCIA: ¿QUIÉN HABLA EN CLASE?}

Como señalé al inicio de este trabajo, mi meta aquí no es relizar una propuesta didáctica ni metodológica. Sería la tarea de los especialistas en didáctica y pedagogía diseñar estrategias guiadas por directrices y metodologías específicas con el fin de integrar destrezas y contenido en los programas de español en la enseñanza superior.

Aún así, pienso que quizás una de las maneras de conseguir una integración óptima de destrezas y contenidos a nivel universitario sería crear cursos donde en las clases se utilizaran activamente los marcos metodológicos, críticos y teóricos de dos diferentes campos: el de la didáctica y el de la disciplina específica. Un curso donde el especialista en didáctica ayudara a hacer de las destrezas no solo un medio de aprendizaje, sino también en una herramienta conceptual que trabajara de manera integrada con el estudio y análisis crítico del contenido. Pero inmediatamente resulta obvio que integrar en un mismo curso dos campos de estudios diferentes puede resultar problemático. Algunos de los retos más obvios que se presentan son 


\section{Nordic Journal of Modern Language Methodology}

de tipo administrativo y de presupuesto. Estas dificultades pueden ser determinantes a la hora de planificar y dar un curso integrado ya que provocan las siguientes preguntas: ¿Se necesitarán dos profesores en clase a la vez y por tanto el coste del curso se doblará en cuanto a salario? ¿Cómo se administraría la evaluación del aprendizaje de los estudiantes? ¿Es la función de los profesores de didáctica y pedagogía no solo formar futuros profesores, sino también formar a sus colegas de manera contínua? Haciendo referencia al título de esta sección, ¿quién habla en clase?, no cabe duda de que quien debe hablar es el estudiante, pero para la administración, que no siempre ve las cosas como los profesores, quien habla en clase es el profesor y estas preguntas pueden ser cruciales a la hora de conceder o no apoyo para desarrollar un curriculum que verdaderamente tenga integrado un componente utilitario con vistas al futuro laboral de los estudiantes.

El lector ya se habrá dado cuenta de que no soy experta en didáctica. Mi especialización es la literatura hispanoamericana y ha sido mi propia práctica docente la que me ha llevado a articular las preocupaciones que aquí presento. En mis clases de literatura a estudiantes noruegos me enfrento a diario con los textos analizando no solo temas literarios, acercamientos teóricos y contextos culturales, sino también la estructura lingüística del texto en sí: el tono de la escritura, el vocabulario utilizado, las variaciones semánticas del mismo e, incluso, la sintaxis de las oraciones. En otras palabras, observando en detalle lo que se podrían llamar las destrezas lingüísticas de la literatura. Al realizar este tipo de lectura detallada con mis estudiantes, descubro siempre que sus destrezas en español varían: en clase suele haber desde estudiantes que son hispanohablantes hasta los que se registraron en el programa porque han pasado unos meses viajando por Latinoamérica o España, pero que nunca han tomado ningún curso de español. Por ello, muchas veces he deseado tener junto a mi a una especilista en didáctica en clase, pues a pesar de que tengo un interés personal en este campo, no faltan las veces en las que he dudado de mis conocimientos y de mi práctica docente. ¿Conozco y aplico coherentemente una metodología didáctica al contenido que quiero mediar? ¿Cómo promuevo las destrezas necesarias en el estudiante para que acceda a una lectura productiva del texto? ¿Me encuentro, como argüiría Bruton, ante una situación docente no lo suficientemente selecta para la implementación existosa de un acercamiento CLIL?

Como un ejemplo, entre otros, pienso en los retos a los que me enfrento cuando enseño el cuento "Nos han dado la tierra" (1953) de Juan Rulfo, un texto de lectura casi 


\section{Nordic Journal of Modern Language Methodology}

obligatoria para cualquier estudiante que esté interesado en la literatura latinoamericana y que a menudo aparece en los temarios de los cursos introductorios de literatura en español. Se trata de un cuento bastante corto y de una lectura que no parecería ofrecer demasiadas trabas para los estudiantes de primer año, pero cuya interpretación depende en alto grado de un análisis sintáctico y semántico de las estructuras impersonales que en él abundan. ¿Son mis referencias al se impersonal descifrables? ¿Logran mis estudiantes asimilar la importancia que todas las oraciones sin sujeto explícito tienen para el análisis del cuento? ¿Motivan este tipo de retos cognitivos a los estudiantes, tal y como sugieren Lightbown y Spada, o se convierten en frenos y frustraciones en su proceso de aprendizaje? En casos como este, un acercamiento holístico y riguroso a mi enseñanza demanda el tipo de colaboración antes propuesta: en diálogo con mis colegas especialistas en didáctica del español podría crear actividades que aseguraran que los estudiantes pudieran manejar diestramente diferentes formas comunicativas de impersonalidad en español. Y no me refiero al análisis de las estructuras lingüísticas, sino a una competencia comunicativa y práctica. Pues una de las competencias claramente utilitarias y transferibles que mis estudiantes pueden refinar al analizar el cuento de Rulfo, es la manera en que la agencia se puede ocultar o hacer explícita en español.

Son escenarios como este los que me han hecho pensar que quizás nos encontremos ante un cambio epistemológico que nos fuerce a redefinir no solo los programas de estudios, sino también la preparación académica y el campo de investigación de los profesores universitarios de lengua extranjeras: ¿Es suficiente formación un curso estándar de didáctica universitaria que acompañe la carrera de especialización investigadora del docente? ¿Deberíamos todos entrenarnos en acercamientos CLIL para poder dar clases en los programas de español en la enseñanza superior? La cuestión es difícil, y no solo desde un punto de vista administrativo, pues, por un lado, el profesor universitario basa sus clases en su investigación, para lo que ha de estar al día dentro de su campo; pero, por otro, sus clases son y llevan tiempo ya siéndolo, clases en las que se integran destrezas y contenido, aunque no sea de manera muy consciente por parte del docente.

Por ende, como Ena Harrop ya ha señalado, todavía hace falta una renovación teórica, metodológica y administrativa para superar las dificultades hasta ahora mencionadas en la integración de destrezas y contenido: 


\section{Nordic Journal of Modern Language Methodology}

To resolve the tension between content and form, two different measures are needed. Firstly, a better theoretical model for the integration of content and form in CLIL needs to underpin successful practice. This model could also provide the basis for a better coordination of CLIL and foreign language lessons, integrating the linguistic dimension of CLIL and the foreign language lessons in one curriculum ... A second measure to better balance content and language would be to establish what linguistic outcomes are reasonably to be expected of CLIL programme. (60-61)

Lo importante es entender que aunque la integración de destrezas y contenido tiene el potencial de maximizar el aprendizaje del estudiante tanto en su competencia lingüística como en sus conocimientos de contenido y reflexión, esto no ocurre por el mero hecho de "bañar" al estudiante en la lengua extranjera. Como profesores de materias impartidas en español a nivel universitario estamos acostumbrados a reflexionar sobre nuestra práctica docente, pero las propuestas CLIL nos hacen también pensar sobre nuestra competencia académica.

No cabe duda de que para poder implementar un acercamiento de integración de destrezas y contenido a nivel universitario se necesita una infraestructura administrativa que posibilite la colaboración entre docentes de distintas áreas para la creación de cursos y actividades en las que se integren diferentes áreas de conocimiento y especialización. Pero además se necesitan recursos metodológicos específicos para los programas en los que la lengua no solo sea un medio para el aprendizaje, sino también un objeto de estudio y reflexión.

\section{CONCLUSIONES}

Estas breves reflexiones han buscado llamar la atención sobre la necesidad de reconsiderar el papel que la integración de destrezas y contenido juega en los programas de español en las universidades, donde la docencia se imparte en esta lengua. Puede que nos encontremos a las puertas de un cambio que nos fuerce a redefinir la preparación académica y el campo de investigación de los profesores universitarios de lenguas extranjeras. La cuestión es complicada, pues está relacionada con presupuestos, políticas universitarias y decisiones gubernamentales. ¿Es necesario un cambio drástico en la manera en que pensamos nuestros 


\section{Nordic Journal of Modern Language Methodology}

cursos? ¿Cómo influirían los posibles cambios en la definición de las diferentes áreas de especialización? Mi esperanza es que los especialistas en didáctica y pedagogía ayuden a generar e impulsen nuevas propuestas docentes para los programas universitarios de lenguas extranjeras que respondan a los retos y exigencias a los que actualmente se están enfrentando las humanidades.

\section{OBRAS CITADAS}

Baetens-Beardsmore, Hugo, ed. European Models of Bilingual Education. Clevedon: Multilingual Matters, 1993.

Brinton, Donna, Marguerite Ann Snow y Marjorie B. Wesche. Content-Based Second Language Instruction. Boston: Heinle and Heinle, 1989.

Bruton, Anthony. "Is CLIL so beneficial, or just selective? Re-evaluating some of the research." System 39 (2011): 523-532.

Chaput, Patricia P. "Revitalizing the Traditional Program." Language and Content: Discipline- and Content-Based Learning. Merle Kreuger y Frank Ryan, eds. Lexington: D.C. Health, 1993. 148-157.

Crandall, Jodi y G. Richard Tucker. "Content-Based Instruction in Second and Foreign Languages." Language Teaching Methodology for the Nineties. S. Anivan, ed. Singapore: SAEMEO Regional Language Centre, 1990, 83-96.

Cummins, James. “The Role of Primary Language Development in Promoting Educational Success for Language Minority Students.” Schooling and Language Minority Students. A Theoretical Framework. Los Angeles: California State University, 1981. 3-49.

Eide, Liv. Representasjoner av målspråksområdet i fremmedspråket. En studie av Latin-

Amerika i laereb $\phi k e r$ i spansk. Doktorgradsavhandling, Universitetet i Bergen, 2012. European Commission Directorate-General For Education And Culture. Content and Language Integrated Learning (CLIL) at School in Europe. European Union, 2006. ---, Marco estratégico: Educación y Formación 2020. European Union, 2009.

European Parlament, The Council, The Europan Economic and Social Committee and the Committee of the Regions. Rethinking Education: Inventing in Skills for Better SocioEconomic Outcomes. European Union, 2012. 


\section{Nordic Journal of Modern Language Methodology}

Genese, Fred. Integrating language and content: Lessons from immersion. Santa Cruz, CA:

National Center for Research on Cultural Diversity and Second Language Learning, 1994.

Harrop, Ena. "Content and Language Integrated Learning (CLIL): Limitations and

Possibilities." Encuentro. Revista de investigación e innovación en la clase de idiomas.

21 (2012): 57-70.

Ioannou Georgiou, Sophie. "Reviewing the puzzle of CLIL." ELT Journal 66.4 (2012): 495504.

Krashen, Stephen. The Input Hypothesis: Issues and Implications. London: Longman, 1985.

Kunnskapsdepartementet. Meld. St. 25 (2016-2017) - Humaniora i Norge. Erna Solbergs regjering, 2017.

Lightbown, Patsy M. y Nina Spada. How Languages are Learned. Oxford: Oxford UP, 2006. Madrid, Daniel y Elena García Sánchez. "Content-based Second Language Teaching." Present and Future Trends in TEFL. Elena García Sánchez, ed. Almería: Secretariado de Publicaciones de la Universidad de Almería, 2001. 101-134.

Marsh, Daniel, Anne Maljers y Aini-KriistinaHartiala, eds. Profiling European CLIL Classrooms. Jyväskylä: UniCOM, 2001.

Met, Myriam. "Learning language through content: Learning content through language." Foreign Language Annals, 24:4 (1991), 281-295.

Montrul, Silvina. El bilingüismo en el mundo hispanohablante. Chichester: Wiley-Blackwell, 2012.

Olsbu, Inger. "The Future of Literary Texts in the L3 Classroom.” Acta Didactica Norge Tidskrift for fagdidaktisk forsknings- og utviklingsarbeid i Norge, 8:2 (2014), Art. 10, 118.

Olsbu, Inger y Kari Soriano Salkjelsvik. “Objetos perdidos: La literatura en la clase E/LE. Europa y Noruega." Hispania, 91:4 (2008), 865-876.

Pérez Gras, María Laura. "La utopía de una enseñanza bilingüe destinada al aborigen en la argentina del siglo XIX." Revista Historia de la Educación Latinoamericana [en linea] 2010, 15 ( ). 